Como citar este artículo: Giraldo-Ospina B, Ramírez-Hoyos LS, Henao-Nieto DE, Flórez-Salazar M, Parra-Londoño F, Gómez-Giraldo EL, Mantilla-Moreno OJ. Estimación de la prevalencia de parásitos intestinales en niños de dos comunidades colombianas. Revista Biosalud 2015; 14(2):19-28. DOI: 10.17151/biosa.2015.14.2.3

\title{
ESTIMACIÓN DE LA PREVALENCIA DE PARÁSITOS INTESTINALES EN NIÑOS DE DOS COMUNIDADES COLOMBIANAS'
}

\author{
Beatriz Giraldo-Ospina² \\ Luz Stella Ramírez-Hoyos ${ }^{3}$ \\ Daniel Eduardo Henao-Nieto ${ }^{4}$ \\ Mateo Flórez-Salazar ${ }^{5}$ \\ Fernando Parra-Londoño ${ }^{6}$ \\ Ermin Leandro Gómez-Giraldo ${ }^{7}$ \\ Omar Jahir Mantilla-Moreno ${ }^{8}$
}

\section{RESUMEN}

Objetivo: Determinar la presencia de enteroparásitos en niños de edad preescolar y escolar en dos comunidades de Risaralda (Colombia). Metodología: Se trata de un estudio de estimación de prevalencia -corte transversalusando una muestra de 258 niños, seleccionados de forma no aleatoria a través de una actividad de demanda inducida. De cada niño se obtuvieron muestras de heces que fueron procesadas mediante la técnica de Ritchie. El método de Graham se aplicó en menores que refiriesen prurito anal. Además, por parte de los padres de familia, se diligenció una encuesta para indagar acerca de las condiciones clínicas en relación con el parasitismo intestinal. Resultados: La prevalencia general de enteroparásitos fue del $37,2 \%$. Los casos de monoinfección e infección mixta se presentaron en el $28,7 \%$ y 2,3\%, respectivamente. Los enteroparásitos patógenos encontrados según su frecuencia fueron: Blastocystis hominis 16,7\%, complejo Entamoeba histolytica/dispar 8,9\%, Giardia lamblia 7\%, Enterobius vermiculares 0,8\%. Además, se hallaron otros enteroparásitos no patógenos. La infección fue más frecuente en hombres. Conclusión: Se identificó una prevalencia general y específica por cada microorganismo de aproximadamente la mitad a la encontrada en otros estudios de similares características. Los niños evaluados presentan factores de alto riesgo para dicha infección y, por tanto, para las consecuencias desfavorables que de estos se derivan.

Palabras clave: enfermedades parasitarias, epidemiología, impactos en la salud, comunidades vulnerables.

\footnotetext{
${ }^{1}$ Investigación del Grupo de Investigación en Salud y Comunidad (GISCO) de la Fundación Universitaria Autónoma de las Américas, Pereira, Colombia.

2 Magíster en Microbiología. Profesora investigadora, Fundación Universitaria Autónoma de las Américas. Pereira, Colombia. Autor para correspondencia. Correo electrónico: p_giraldob_154@uam.edu.co

${ }^{3}$ Especialista en Epidemiología. Profesora investigadora, Fundación Universitaria Autónoma de las Américas. Pereira, Colombia. Correo electrónico: luzstellita28@hotmail.com

${ }^{4}$ Ph.D. en Salud Pública y Epidemiología (C). Profesor, Observatorio EcoRegional de Salud Pública, Facultad de Ciencias de la Salud, Universidad Tecnológica de Pereira. Asesor de la Fundación Universitaria Autónoma de las Américas. Pereira, Colombia. Correo electrónico: d.henao@utp.edu.co

5 Estudiante IX semestre. Facultad de Medicina, Fundación Universitaria Autónoma de las Américas. Autor para correspondencia. Pereira, Colombia. Correo electrónico: salazarmateof@hotmail.com

${ }^{6}$ Estudiante IX semestre, Facultad de Medicina, Fundación Universitaria Autónoma de las Américas. Correo electrónico: ferchoaurora@hotmail.com

7 Estudiante IX semestre. Facultad de Medicina, Fundación Universitaria Autónoma de las Américas. Pereira, Colombia. Correo electrónico: erlean0322@hotmail.com

${ }^{8}$ Estudiante IX semestre. Facultad de Medicina, Fundación Universitaria Autónoma de las Américas. Pereira, Colombia. Correo electrónico: tuco8265@hotmail.com
} 


\section{ESTIMATE OF INTESTINAL PARASITES PREVALENCE IN INFANTS OF TWO COLOMBIAN COMMUNITIES}

\section{ABSTRACT}

Objective: To determine the presence of intestinal parasites in preschool and school age children in two communities of Risaralda Department (Colombia). Methodology: Crosssectional study of prevalence estimation using a sample of 258 children selected in a nonrandom sorting through an induced demand activity. Fecal samples were taken from each child and were processed using Ritchie's technique. The Graham method was applied to infants who presented anal itching. In addition, the parents filled out a survey to determine the clinical

\section{INTRODUCCIÓN}

Los parásitos intestinales (PI) aún son un serio problema de salud pública (1-3). Su importancia viene derivada tanto por sus altas frecuencias relativas de ocurrencia como por las características de la población que actualmente los sufre. Los riesgos para sufrir una parasitosis son de dos tipos: inmodificables y modificables. Dentro de los primeros, la edad es el más importante y los niños, por su inmadurez inmunológica, son más susceptibles. Pero son los segundos, los que se pueden catalogar como sociales (y de higiene pública), los que son determinantes y además intervenibles -ellos son, por ejemplo, el acceso adecuado a agua potable y la buena disposición de excretas-.

Las parasitosis intestinales causadas por ejemplo por el complejo Entamoeba histolytica/ dispar y la Giardia lamblia se encuentran entre las 10 infecciones más comunes observadas en el mundo; afectando aproximadamente a 3500 conditions related to intestinal parasitism. Results: The general prevalence of enteric parasites was $37.2 \%$. Infections by a single or mixed infection were present in $28.7 \%$ and $2.3 \%$ of the population respectively. The pathogenic enteric parasites found were: Blastocystis hominis 16.7\%, Entamoeba histolytica/dispar 8.9\%, Giardia lamblia $7 \%$, and Enterobius vermiculares $0.8 \%$. Likewise, other non-pathogen enteric parasites were found and the infection was more frequent in males. Conclusion: General and specific prevalence for each organism approximately half of that found in other similar studies of simple characteristics was identified. The children evaluated present high risk factors for intestinal parasite infection and, therefore, to suffer the unfavorable consequences derived it.

Key words: parasitic disease, epidemiology, impact on health, vulnerable populations.

millones de personas y produciendo cada año entre 40 y 110 mil muertes. La E. histolytica, el agente causal de la amebiasis, provoca enfermedad severa en 48 millones de personas y mata todos los años alrededor de $70 \mathrm{mil}$ individuos. Del mismo modo, se determinó que 13 y 33 millones de niños preescolares y escolares respectivamente, tienen riesgo de contraer infecciones por parásitos intestinales (4-6).

La situación mundial de la parasitosis es heterogénea: en los países ricos su existencia es prácticamente nula y en los pobres su prevalencia es excesivamente alta. De lo anterior se puede inferir entonces, que la frecuencia de ocurrencia de las parasitosis está asociada a la pobreza, siendo así, un marcador de desarrollo. Para Latinoamérica y el Caribe esta situación es problemática porque la ocurrencia del problema se ha mantenido inalterada por más de 60 años.

Colombia no es ajeno a esta realidad, siendo uno de los 17 países con mayor número de niños en 
edad preescolar y escolar en riesgo de infección por parásitos intestinales (7). En la región del Eje Cafetero (Centro del país), específicamente en Dosquebradas (Risaralda), espacio-población donde se llevó a cabo el presente estudio, se conocen muy pocos estudios que relacionen la prevalencia de los enteroparásitos. Sin embargo, en regiones cercanas pertenecientes al departamento del Quindío, se reportan altas prevalencias de protozoarios como Blastocystis hominis, enteroparásito emergente $(5,8-11)$ que ha tomado gran interés por su controversia en términos de patogenicidad e incremento en la prevalencia durante los últimos años (8). De igual manera, en la región de Cundinamarca y Boyacá se reportan elevadas prevalencias de los mismos parásitos patógenos en poblaciones escolares y adolescentes (12).

En los últimos años los parasitosis han cambiado su curso clásico con el mejoramiento de las medidas sanitarias. Los parásitos macroscópicos se hanido erradicando como causa de enfermedad intestinal y los protozoos han ido aumentando significativamente. Además, han surgido otros elementos parasitarios denominados parásitos emergentes comúnmente pesquisados como Blastocystis sp., los cuales han producido un cambio conceptual de aplicación práctica en la epidemiología y patogenia, síndrome clínico y tratamiento de las enfermedades $(8,13,14)$. Sin embargo, aún persiste este problema y por eso es indispensable moldearlo empíricamente, sobre todo, en zonas de habitación de población vulnerable.

La población y, dentro de ella, las comunidades con las que se compartieron experiencias académicas y de desarrollo social, se encontraron en el departamento de Risaralda -específicamente en el municipio de Dosquebradas-. Las dos comunidades se pueden clasificar como vulnerables, de acuerdo con su alta proporción de hogares con más de tres necesidades básicas insatisfechas. Allí, tanto la Fundación Universitaria Autónoma de las Américas como la Secretaría de Salud de Risaralda, llevan a cabo actividades orientadas a la construcción de capacidades para la autogestión de problemas y el desarrollo de valor social. Dentro del cuerpo de todas las actividades llevadas a cabo se adelantó una búsqueda activa de potenciales casos de parasitosis intestinal mediante el análisis microscópico de heces implementando la técnica de Ritchie, frotis rectal por el método de Graham y el diligenciamiento de una encuesta acerca de condiciones clínicas en torno a dicha condición, en niños de 1 a 10 años de edad adscritos a controles de crecimiento y desarrollo en las comunidades de La Badea y Frailes, del municipio de Dosquebradas (Risaralda, Colombia).

\section{MATERIALES Y MÉTODOS}

El presente estudio es descriptivo transversal. Se realizó en los sectores de La Badea y Frailes, del municipio de Dosquebradas (Risaralda, Colombia), acompañados por la Fundación Universitaria Autónoma de las Américas en sus prácticas académicas de promoción de la salud y detección temprana y protección específica de la enfermedad, durante el segundo semestre de 2012 y el primer semestre de 2013.

Es importante aclarar que el muestreo no se realizó de forma aleatoria sino que los niños incluidos en el estudio fueron parte de un programa de demanda inducida y búsqueda activa de niños en riesgo, que realizó la Fundación Universitaria Autónoma de las Américas, como parte integral de sus prácticas académicas, y que se enmarcaron en el Plan de Intervenciones Colectivas, definido por el Ministerio de Salud y la Protección Social en la Ley 1438 de 2011 y la Resolución 0518 de 2015.

Dentro de la descripción de la población se puede decir que el municipio de Dosquebradas tiene 173.452 habitantes (15), que a su vez hacen parte de los 859.666 del departamento de Risaralda (16), según el censo de 2005 realizado por el Departamento Administrativo Nacional de Estadísticas (DANE). 
Consecuentemente, se tomó una muestra -por conveniencia como ya se advirtió- de 258 niños entre 1 y 10 años de edad adscritos a controles de crecimiento y desarrollo, un programa de vigilancia de los indicadores antropométricos y de desarrollo psicosocial de la primera infancia, que actualmente es política del Estado colombiano. Se incluyeron un total de 258 niños participantes de forma voluntaria en el estudio, luego de diligenciar el consentimiento informado por parte de sus padres o representante legal y el asentimiento informado en aquellos niños que fuesen mayores de 5 años. Se excluyeron los niños con tratamiento antiparasitario realizado en los últimos 6 meses o durante el estudio, o quienes tuviesen una edad menor de 1 año o mayor de 10 años y/o no residieran en las comunidades mencionadas.

Se brindó a los padres de familia, previamente captados por enfermeras, profesoras y madres comunitarias, una charla informativa sobre el parasitismo intestinal y se les invitó a participar en el estudio, en donde se les explicó con claridad cada procedimiento y luego se categorizó el trabajo como investigación de riesgo mínimo (17). Luego de aceptar la participación, se les realizó una encuesta acerca de las condiciones clínicas de cada niño y se diligenció el consentimiento y asentimiento informado siguiendo las normas éticas para trabajos en la comunidad estipuladas en la Resolución 008430 de 1993 del Ministerio de Salud y Protección Social de Colombia (17).

Para la obtención de las muestras se suministró un frasco para el almacenamiento de la materia fecal, dichos especímenes fueron refrigerados. Las muestras se procesaron en el Laboratorio de Fundamentación de la Fundación Universitaria Autónoma de las Américas; a cada coprológico se le realizó el método de concentración de formol éter (11), además frotis fecal directo con suero salino y solución yodada $(18,19)$, el análisis de las muestras solo se realizó microscópicamente.

Los niños que presentaron prurito anal se sometieron voluntariamente -mediante consentimiento informado diligenciado por sus padres o representante legal- a una toma de muestra de la región perianal en horas de la mañana y sin previo aseo, para el diagnóstico de Enterobius vermiculares mediante método de la cinta engomada o de Graham según especificaciones de Botero y Restrepo (19).

El estudio fue avalado por el Comité Científico del Hospital Santa Mónica de Dosquebradas y el Comité de Ética del Departamento de Investigaciones de la Fundación Universitaria Autónoma de las Américas. La identidad de los sujetos de estudio se mantiene en confidencialidad, lo cual consta en los archivos institucionales. Para la aplicación del tratamiento se direccionó cada uno de los niños parasitados al Hospital Santa Mónica de Dosquebradas, quien garantizó el tratamiento de acuerdo con el parásito encontrado.

Los datos obtenidos en la aplicación de las encuestas y el procesamiento de las muestras de materia fecal fueron organizados en tablas electrónicas del programa Prism 5 (GraphPad). Las variables fueron resumidas de acuerdo con su naturaleza -medias para variables continuas; proporciones y razones de proporción para variables categóricas-, y los procedimientos de inferencia causal se llevaron a cabo usando las pruebas de hipótesis respetando dicha naturaleza: $\mathrm{t}$ student para comparar medias $\mathrm{y}$ chi-cuadrado para comparar proporciones. El análisis fue elaborado mediante el programa SPSS versión 15 (SPSS Inc., Chicago, USA).

\section{RESULTADOS}

La Tabla 1 presenta la descripción sociodemográfica de la muestra de acuerdo con la comunidad de procedencia, evidenciando un predominio del sexo masculino en la edad preescolar. La edad promedio para ambos sexos en ambas comunidades fue de 4,8 años, con una mediana de 4 años, lo cual sugiere una distribución normal de los datos. 
Tabla 1. Distribución sociodemográfica de la muestra.

\begin{tabular}{lccc}
\hline SEXO/EDAD & FRAILES & LA BADEA & TOTAL \\
\hline FEMENINO & $\mathbf{5 1}$ & $\mathbf{5 9}$ & $\mathbf{1 1 0}$ \\
1-5 años & 24 & 42 & 66 \\
6-10 años & 27 & 17 & 44 \\
MASCULINO & $\mathbf{7 4}$ & $\mathbf{7 4}$ & $\mathbf{1 4 8}$ \\
1-5 años & 47 & 61 & 108 \\
6-10 años & 27 & 13 & 40 \\
TOTAL & $\mathbf{1 2 5}$ & $\mathbf{1 3 3}$ & $\mathbf{2 5 8}$ \\
\hline
\end{tabular}

De un total de 258 niños se identificó una prevalencia de parasitismo intestinal del $37,2 \%$. La Tabla 2 presenta la distribución de frecuencias por parásito hallada en cada una de las comunidades y la relación con las manifestaciones clínicas de cada uno de los niños, evidenciando que la prevalencia más alta fue para Blastocystis sp., seguido por el complejo E. histolytica/dispar y G. lamblia, infecciones que estuvieron en directa relación con la manifestación clínica más frecuente: dolor abdominal. Además, se detectaron otras enteroparasitosis de tipo no patógenas como Iodamoeba bütschlii, Endolimax nana y Entamoeba coli.

Se presentaron 74 casos (28,7\%) de monoinfección y 6 casos de infección mixta, por agentes patógenos exclusivos. La Tabla 3 presenta la prevalencia de parásitos intestinales según el tipo de infección en relación con la edad.

En relación con Blastocystis sp. y su predominante prevalencia, cabe señalar que de $43(100 \%)$ casos, $14(32,5 \%)$ se asociaron a coinfección con $G$. lamblia u otros agentes no patógenos. Esto indica que por cada tres infecciones por el protozoo, una de ellas se encontraba en coinfección con otros agentes parasitarios. De igual manera, debe resaltarse que su preponderante prevalencia se relaciona directamente con las manifestaciones clínicas, evidenciando que estas se presentan con más frecuencia en aquellos menores infectados con Blastocystis sp.
Las principales manifestaciones clínicas reportadas en los menores infectados por cualquier agente parasitario fueron: dolor abdominal $(19,8 \%)$, prurito anal $(12,4 \%)$, pérdida de peso $(7,4 \%)$, vómitos $(5 \%)$, diarreas frecuentes $(4,3 \%)$, deposición con moco o sangre $(2 \%)$, entre otros. Solo el 3,9\% de los casos infectados negaron tener alguna manifestación clínica.

Únicamente se detectaron dos casos con helmintos -mediante el test de Graham- en este estudio, revelado en la Tabla 2; uno de ellos presenta infección mixta con complejo $E$. histolytica/dispar y I. bütschlii.

\section{DISCUSIÓN}

Los resultados muestran una prevalencia de parásitos intestinales del $37,2 \%$, con un particular predominio de protozoos y en especial de Blastocystis sp. el más prevalente $(16,7 \%)$. Este resultado coincide con múltiples estudios, los cuales lo ubican como el protozoo más prevalente, hegemonía que ha despertado una controversia reciente sobre su posible rol patógeno (8), dada su capacidad de producir manifestaciones clínicas relevantes. Un estudio presentado en el año 1989 con 19.252 muestras de heces, reporta la presencia exclusiva de Blastocystis sp. en 107 de 239 pacientes que presentaron síntomas, y señala que los 132 restantes cursaban con el protozoo en coinfección por enteroparásitos no patógenos; en el mismo estudio, concluyen que el Blastocystis sp. debe ser considerado patógeno (20). 


\section{Biosalud}

Beatriz Giraldo-Ospina et al.

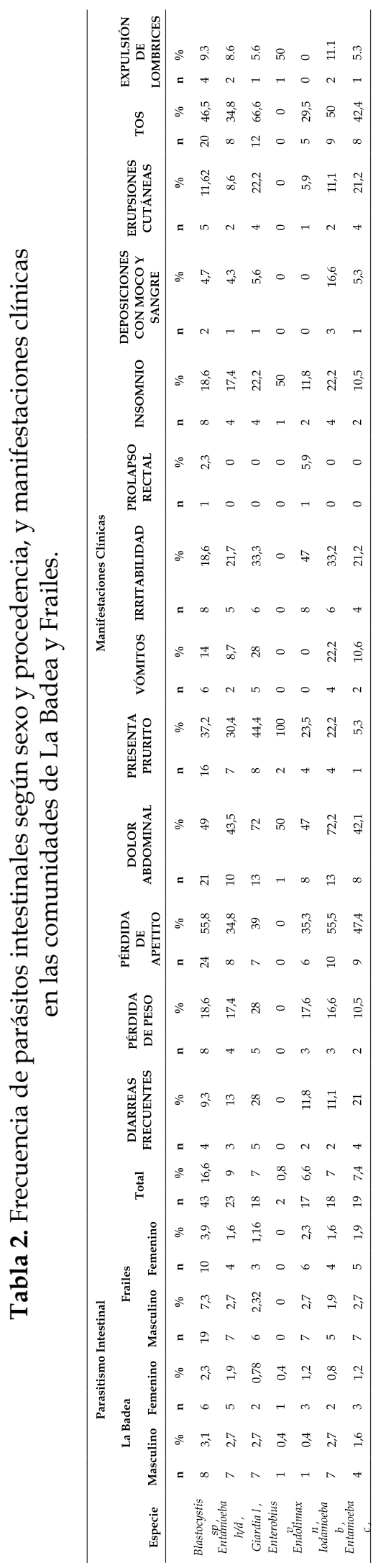


Tabla 3. Prevalencia de parásitos intestinales según tipo de infección y edad.

\begin{tabular}{lllllll}
\hline Edad & \multicolumn{2}{l}{ Monoparásitos } & \multicolumn{2}{l}{ Poliparásitos } & \multicolumn{2}{c}{ Total } \\
\hline $\begin{array}{l}\text { Preescolares } \\
\text { (1-5 años) }\end{array}$ & $\mathbf{\mathbf { n } ^ { \mathbf { 0 } }}$ & $\mathbf{0}$ & $\mathbf{n}^{\mathbf{0}}$ & $\%$ & $\mathbf{n}^{\mathbf{0}}$ & $\%$ \\
$\begin{array}{l}\text { Escolares } \\
\text { (6-10 años) }\end{array}$ & 24 & 13,2 & 23 & 8,9 & 57 & 22,1 \\
Total & 58 & 9,3 & 15 & 5,8 & 39 & 15,1 \\
\hline
\end{tabular}

Un estudio realizado en la zona urbana de Calarcá, municipio que se encuentra en la misma región geográfica que Dosquebradas (Cordillera Central de los Andes), presentó resultados que coinciden con el orden de frecuencias de cada enteroparásito según lo muestra la Tabla 4 (10). En forma similar, un estudio realizado en Circasia, municipio aledaño a la zona de estudio (9), presenta una prevalencia determinada por los mismos tres protozoos hallados en el estudio de Calarcá y en el presente informe revelado en la Tabla 4. De otra parte y al margen de la región abordada, se encuentra que el departamento de Boyacá en su capital, Tunja, reporta un particular predominio de Blastocystis sp. $(67,9 \%)$, seguido por E. histolytica/dispar (8,7\%), G. Lamblia $(7,8 \%)$ y otros enteroparásitos (12).

Por otra parte, Martínez-Barbabosa et al. encontraron una alta presencia de Blastocystis sp. con un $80 \%$ de prevalencia (14). De la misma manera, un estudio realizado en Maracaibo (Venezuela) indica una prevalencia del $80 \%$ para Blastocystis sp. seguido de otras especies como E. histolytica/dispar, G. Lamblia y enteroparásitos no patógenos (21). Y otro estudio realizado en Venezuela indica que casi 2 de cada 3 niños $(62,6 \%)$ se encontraban parasitados por Blastocystis sp. $(44,6 \%)$, además de G. lamblia $(12,6 \%)$ y otras especies de helmintos o enteroparásitos no patógenos (22).

El presente estudio indicó una muy baja prevalencia de helmintos con 2 muestras positivas para E. vermiculares $(2,3 \%)$, dato que se relaciona con algunos estudios que reportan prevalencias similares a las de Marcano et al. con únicamente 2 casos de helmintiasis por el mismo patógeno (2), Londoño et al. con solo 2 casos determinados por A. lumbricoides (10) y otros estudios donde predominaron los protozoos $(6,23,24)$.

A partir del análisis realizado en el presente estudio, se identifica que la comunidad intervenida presenta una prevalencia general y específica por cada microorganismo de aproximadamente la mitad con respecto a otros estudios relacionados en la Tabla 4 (9-11, 22, 2528), lo cual da algunos indicios sobre la posible efectividad en el acompañamiento acerca de temas de promoción, prevención y hábitos de vida saludables por parte de la Fundación Universitaria Autónoma de las Américas a partir de 2011 en dichas comunidades. El impacto de la presencia de la Universidad en la comunidad es tema actual de discusión y será objetivo de futuros trabajos de investigación.

Debido a la naturaleza -descriptiva- del estudio se puede afirmar que la caracterización planteada -a través de prevalencias y razones de prevalencia- le saca el mayor beneficio a la información recolectada. Los ejercicios de pruebas de hipótesis, como medidas de inferencia causal (que permite identificar posibles factores de riesgos/protectores asociados al desenlace) serán objetivo de futuros proyectos diseñados idóneamente (por ejemplo, estudios de series de tiempo interrumpidas) para ello. El principal sesgo del presente trabajo es el de selección, ya que la muestra no fue aleatoria; sin embargo, las características generales de la muestra se acomodan a los registros demográficos y a otras muestras similares, por lo que se sospecha que el efecto de esta sobre los resultados fue poco. 
Beatriz Giraldo-Ospina et al.

Tabla 4. Razones de prevalencia con base a otros estudios. 19

\begin{tabular}{|c|c|c|c|c|c|}
\hline Fuente & General & $\begin{array}{c}\text { B. } \\
\text { hominis }\end{array}$ & E. histolytica/dispar & $\begin{array}{c}G . \\
\text { lamblia }\end{array}$ & $\begin{array}{c}\text { Muestra } \\
(\mathrm{n}=\text { niños })\end{array}$ \\
\hline Comunidades estudiadas & $32,5 \%$ & $16,7 \%$ & $8,9 \%$ & $7 \%$ & 258 \\
\hline Calarcá, Colombia (10) & $53,8 \%$ & $36,5 \%$ & $10,9 \%$ & $13,2 \%$ & 220 \\
\hline Razón de prevalencia & 1,7 & 2,2 & 1,2 & 1,9 & - \\
\hline Circasia, Colombia (9) & - & $57 \%$ & $3,8 \%$ & $18,2 \%$ & 79 \\
\hline Razón de prevalencia & - & 3,4 & 0,4 & 2,6 & - \\
\hline Armenia, Colombia (11) & - & $6,1 \%$ & $9,1 \%$ & $12,8 \%$ & 328 \\
\hline Razón de prevalencia & - & 0,4 & 1 & 1,8 & - \\
\hline Sabaneta, Colombia (25) & $81,4 \%$ & $1 \%$ & $27,8 \%$ & $24,7 \%$ & 97 \\
\hline Razón de prevalencia & 2,5 & 0,1 & 3,1 & 3,5 & - \\
\hline Bolívar, Venezuela (22) & $62,6 \%$ & $44,6 \%$ & - & $12,6 \%$ & 278 \\
\hline Razón de prevalencia & 1,9 & 2,7 & - & 1,8 & - \\
\hline Mendoza, Argentina (26) & - & $54,9 \%$ & - & $13,1 \%$ & 221 \\
\hline Razón de prevalencia & - & 3,3 & - & 1,9 & - \\
\hline Costa Atlántica, Colombia (27) & $92 \%$ & $29 \%$ & $54 \%$ & $17 \%$ & 381 \\
\hline Razón de prevalencia & 2,8 & 1,7 & 6,1 & 2,4 & - \\
\hline Minas Gerais, Brasil (28) & $29,3 \%$ & - & $3,8 \%$ & $19,2 \%$ & 133 \\
\hline Razón de prevalencia & 0,9 & - & 0,4 & 2,7 & - \\
\hline $\begin{array}{l}\text { Promedio de razones de prevalencia } \\
\text { por parásito en Frailes }\end{array}$ & 1,6 & 1,4 & 2,1 & 2,3 & 125 \\
\hline $\begin{array}{l}\text { Promedio de razones de prevalencia } \\
\text { por parásito en La Badea }\end{array}$ & 2,3 & 3,1 & 2 & 2,4 & 133 \\
\hline $\begin{array}{l}\text { Promedio de razones de prevalencia } \\
\text { general }\end{array}$ & 1,96 & 1,96 & 2,05 & 2,34 & - \\
\hline
\end{tabular}

Una dificultad identificada en el desarrollo de la investigación estuvo relacionada con la poca disposición de donar materia fecal, dado que de los 1.200 recipientes para recolección repartidos en la comunidad, únicamente se logró recolectar el $22 \%$.

Es importante reconocer las limitaciones del estudio en tanto no es posible estimar precisamente el número de niños reales que habitan en las dos comunidades. Este es un sesgo de la investigación que debe reconocerse; sin embargo no debe invalidar el alcance de los resultados si se tiene en cuenta que ni siquiera los censos oficiales del Estado (a través del DANE) tienen una cifra precisa. Por lo tanto, debe considerarse este esfuerzo como un pilotaje a partir del cual podrán hacerse estudios más complejos y estadísticamente más precisos.

Si bien la prevalencia encontrada en esta investigación es menor a la prevalencia encontrada en otros estudios de similares características metodológicas tal como se

${ }^{9}$ La Tabla 4 presenta la relación entre la prevalencia encontrada por parásito en otras investigaciones y la prevalencia por parásito hallada en la presente investigación. Se promediaron los valores hallados por cada parásito y, en términos generales, se evidencia que (para los cuatro parásitos patógenos) dichas razones oscilan entre 1,96-2,34 por lo cual se estableció que las comunidades del presente estudio tienen la mitad de la prevalencia en relación con otros estudios de características sociodemográficas y metodológicas similares y/o iguales. 
plantean en Tabla 4, es importante entender que ello es reflejo de las condiciones de higiene y saneamiento inadecuado en las que dichas comunidades viven. Por otro lado, la baja frecuencia de helmintos con respecto a protozoos desdibuja la utilidad real -basados en el contextode la desparasitación masiva determinada por la OMS con dosis única de albendazol. Finalmente, se recomienda la vigilancia periódica de parásitos intestinales por técnicas idóneas para ello (18), teniendo en cuenta que las cifras del presente estudio se podrían aproximar a que uno de cada tres menores se encontraba parasitado por algún agente y que nueve de cada 10 menores refirieron alguna sintomatología.

\section{AGRADECIMIENTOS}

ARicardo de Jesús Jiménez-Mejía, Rector ejecutivo de la Fundación Universitaria Autónoma de las Américas y Decano de la Facultad de
Medicina, por su incondicional apoyo logístico y administrativo. A Catalina López-Salazar por sus traducciones. A la Fundación Universitaria Autónoma de las Américas y al Hospital Santa Mónica de Dosquebradas por su apoyo, financiamiento y su propuesta de promoción de la salud y prevención de la enfermedad en las comunidades vulnerables de la región.

\section{FINANCIACIÓN}

La investigación fue financiada por la Fundación Universitaria Autónoma de las Américas y el Hospital Santa Mónica de Dosquebradas.

\section{CONFLICTO DE INTERESES}

Los autores declaran no tener ningún conflicto de intereses.

\section{REFERENCIAS}

1. Sánchez L. Una mirada a las enfermedades parasitarias en el país. NOVA - Publicación Científica 2006; 4(5):100-103.

2. Marcano Y, Suárez B, González M, Gallego L, Hernández T, Naranjo M. Caracterización epidemiológica de parasitosis intestinales en la comunidad 18 de Mayo, Santa Rita, estado Aragua, Venezuela. Bol Mal Salud Amb 2012; 53(2):135-145.

3. Ávila H, Ávila A, Araújo J, Villarreal A, Douglas T. Associated factors for intestinal parasitosis in children in an outpatient clinic. Rev Mex Pediatr 2007; 74(1):5-8.

4. Lay $T$, Montalván $B$, Eléspuru $A$, Vásquez $J$, Hemeryth $R$, Ríos $M$, et al. Incidencia de parásitos intestinales y estado nutricional en niños preescolares de la I.E.I. No 165 "República Federal de Alemania" Punchana 2008-2009. Disponible en: http://www.unapiquitos.edu.pe/investigacion/oginv/ descargas/2009/Art.Cientifico\%20Blga.\%20Tania\%20Lay.pdf

5. Santana EC. La parasitosis intestinal. Un serio problema médico-social Revisión Bibliográfica. Revista Electrónica de Portales Médicos 2009; 1-15.

6. Corrales L, Hernández S, Rodríguez MA, Hernández A. Parasitismo intestinal infantil: factores epidemiológicos en Orange Walk, Belice. Rev Ciencias Médicas 2011; 15(4):163-178.

7. Organización Panamericana de la Salud, McGill University. Taller sobre la integración de la desparasitación en los paquetes de atención en salud para niños en edad preescolar en las Américas; 2011. Disponible en: http://www.paho.org/hq/index.php?option=com_docman\&task=doc_view\&gid=15255\&Itemid=

8. Rodríguez E, Mateos B, González JC, Aguilar YM, Alarcón E, Mendoza AA, et al. Transición parasitaria a Blastocystis hominis en niños de la zona centro del estado de Guerrero, México. Parasitol Latinoam 2008; 63(1-2-3-4):20-28. 
Beatriz Giraldo-Ospina et al.

9. Arias JA, Guzmán GE, Lora-Suárez FM, Torres E, Gómez JE. Prevalencia de protozoos intestinales en 79 niños de 2 a 5 años de edad de un hogar infantil estatal en Circasia, Quindío. Infect 2010; 14(1):31-38.

10. Londoño AL, Mejía S, Gómez-Marín JE. Prevalencia y factores de riesgo asociados a parasitismo intestinal en preescolares de zona urbana en Calarcá Colombia. Rev Salud Pública 2009; 11(1):7281.

11. Giraldo-Gómez JM, Lora F, Henao LH, Mejía S, Gómez-Marín JE. Prevalencia de Giardiasis y Parásitos Intestinales en prescolares de hogares atendidos en un programa estatal en Armenia, Colombia. Revista de Salud Pública 2005; 7(3):327-338.

12. Manrique-Abril FG, Suescún-Carrero SH. Prevalencia de parasitismo intestinal y situación nutricional en escolares y adolescentes de Tunja. Revista CES Medicina 2011; 25(1):20-30.

13. Vidal S, Toloza L, Cancino B. Evolución de la prevalencia de enteroparasitosis en la ciudad de Talca, Región del Maule, Chile. Rev Chil Infect 2010; 27(4):336-340.

14. Martínez-Barbabosa I, Gutiérrez-Quiroz M, Ruiz-González L, Ruiz-Hernández AL, Gutiérrez-Cárdenas EM, Gaona E. Blastocystis hominis y su relación con el estado nutricional de escolares en una comunidad de la sierra de Huayacocotla, Veracruz, México. Rev Biomed 2010; 21:77-84.

15. Departamento Administrativo Nacional de Estadísticas. Boletín Censo General 2005. Perfil Dosquebaradas-Risaralda. Colombia. 2010; 1-6.

16. Departamento Administrativo Nacional de Estadísticas. Boletín Censo General 2005. Perfil Risaralda. Colombia. 2010; 1-6.

17. Ministerio de Salud. Resolución número 8430 de 1993. Bogotá, República de Colombia; 1993. p. 1-15.

18. Restrepo IC, Mazo LP, Salazar ML, Montoya MN, Botero JG. Evaluación de tres técnicas coproparasitoscópicas para el diagnóstico de geohelmintos intestinales. Iatreia 2013; 26(1):15-24.

19. Botero D, Restrepo M. Parasitosis humanas. Quinta Edición. Medellín: Corporación para Investigaciones Biológicas; 2012. p. 694-709.

20. Qadri SM, Al-Okaili GA, Al-Dayel F. Clinical Significance of Blastocystis hominis. J Clin Microbiol 1989; 27(11):2407-2409.

21. Acurero EM, Calchi La Corte M, Merchán FM, Useche PE. Prevalencia de B. hominis. en preescolares y escolares del municipio Maracaibo, Venezuela. Rev. Soc. Ven. Microbiol 2013; 33(2):146-150.

22. Devera R, Requema I. Prevalencia de parásitos intestinales en escolares de la escuela básica estatal José Félix Blanco, Estado Bolívar, Venezuela. Salus Online 2010; 14(3):43-48.

23. Pérez-Gordon G, Rosales MJ, Valdez RA, Vargas-Vásquez F, Cordova O. Detección de parásitos intestinales en agua y alimentos de Trujillo, Perú. Rev. Perú. Med. Exp. Salud Pública 2008; 25(1):144148.

24. Aher A, Kulkarni S. Prevalence of intestinal parasites in school going children in a rural community. International Journal of Biomedical Research 2011; 2(12): 605-607.

25. Tabares LF, González L. Prevalence of intestinal parasites in children under 12 years of age, hygienic habits, characteristics of the houses and presence of bacteria in the drinking water of a locality of Sabaneta, Colombia. Iatreia 2008; 21(3):253-259.

26. Puente AP. Prevalencia de la infección por Giardia lamblia y otros parásitos intestinales en una población infantil suburbana de la ciudad de Mendoza, Argentina. Parasitol Latinoam 2007; 62(1-2):49-53.

27. Agudelo-López S, Gómez-Rodríguez L, Coronado X, Orozco A, Valencia-Gutiérrez CA, RestrepoBetancur LF, et al. Prevalencia de parasitosis intestinales y factores asociados en un corregimiento de la Costa Atlántica colombiana. Rev. Salud Pública 2008; 10(4):633-42.

28. Ribeiro AL, Belizário TL, Pimentel JdeB, Penatti MP, Pedroso RdosS. Prevalence of intestinal parasites in preschool children in the region of Uberlândia, State of Minas Gerais, Brazil. Revista da Sociedade Brasileira de Medicina Tropical 2011; 44(2):191-193. 